\title{
Retraction Note to: MicroRNA-30c-regulated HDAC9 mediates chemoresistance of breast cancer
}

\author{
Zhongxing Liang $^{1,2} \cdot$ Amber Feng $^{1} \cdot$ Hyunsuk Shim ${ }^{1,2}$
}

Published online: 24 February 2022

๑) Springer-Verlag GmbH Germany, part of Springer Nature 2022

\section{Retraction to: \\ Cancer Chemotherapy and Pharmacology (2020) \\ 85:413-423 \\ https://doi.org/10.1007/s00280-019-04024-9}

The Editors-in-Chief have retracted this article. After publication, concerns were raised about the western blot images presented in Figs. 1, 3 and 4. Specifically:

Repeated background features in "empty" spaces of the western blot gels in Fig. 1a;

Unexpected background changes between western blot bands in Fig. 3b, 4a and 4c.
The corresponding author confirmed that the western blot backgrounds have been altered but stated that the original unedited images were no longer available. The Editors-inChief therefore no longer have confidence in the presented data.

All authors agree to this retraction.

Publisher's Note Springer Nature remains neutral with regard to jurisdictional claims in published maps and institutional affiliations.

The original article can be found online at https://doi.org/10.1007/ s00280-019-04024-9.

Zhongxing Liang

zliang@emory.edu

$\triangle$ Hyunsuk Shim

hshim@emory.edu

1 Department of Radiation Oncology, Emory University, Atlanta, GA 30322, USA

2 Winship Cancer Institute, Emory University, Atlanta, GA 30322, USA 